\title{
Multidrug-resistant gram-negative organisms and association with 1-year mortality, readmission, and length of stay in Veterans with spinal cord injuries and disorders
}

\author{
Swetha Ramanathan ${ }^{1}$ - Margaret A. Fitzpatrick ${ }^{1,2} \cdot$ Katie J. Suda $^{3,4} \cdot$ Stephen P. Burns ${ }^{5,6} \cdot$ Makoto M. Jones ${ }^{7,8}$. \\ Sherri L. LaVela ${ }^{1,9} \cdot$ Charlesnika T. Evans ${ }^{1,10}$
}

Received: 5 August 2019 / Revised: 18 October 2019 / Accepted: 5 November 2019 / Published online: 11 December 2019

(c) The Author(s), under exclusive licence to International Spinal Cord Society 2019

\begin{abstract}
Study design Retrospective cohort study.

Objectives The goal of this study was to assess the impact of multidrug resistant gram-negative organisms (MDRGNOs) on outcomes in those with SCI/D.

Setting VA SCI System of Care, Department of Veterans Affairs, United States.

Methods Multidrug resistance (MDR) was defined as being non-susceptible to $\geq 1$ antibiotic in $\geq 3$ antibiotic classes. Multivariable cluster-adjusted regression models were fit to assess the association of MDRGNOs with 1-year mortality, 30-day readmission, and postculture length of stay (LOS) stratified by case setting patients. Only the first culture per patient during the study period was included.

Results A total of 8,681 individuals with SCI/D had a culture with gram-negative bacteria during the study period, of which $33.0 \%$ had a MDRGNO. Overall, 954 (10.9\%) died within 1 year of culture date. Poisson regression showed that MDR was associated with 1-year mortality among outpatients (IRR: 1.28, 95\% CI, 1.06-1.54) and long-term care patients (OR: 2.06, 95\% CI, 1.28-3.31). MDR significantly impacted postculture LOS in inpatients, as evidenced by a $10 \%$ longer LOS in MDR vs. non-MDR (IRR: 1.10, 95\% CI, 1.02-1.19). MDR was not associated with increased 30-day readmission.
\end{abstract}

Conclusions MDRGNOs are prevalent in SCI/D and MDR may result in poor outcomes. Further attention to prevention of infections, antibiotic stewardship, and management are warranted in this population.

Swetha Ramanathan

swetha.ramanathan@va.gov

1 Center of Innovation for Complex Chronic Healthcare (CINCCH), Department of Veterans Affairs, Edward Hines Jr. VA Hospital, Hines, IL, USA

2 Department of Internal Medicine, Stritch School of Medicine, Loyola University, Chicago, IL, USA

3 Center for Health Equity Research and Promotion, Department of Veterans Affairs, VA Pittsburgh Healthcare System, Pittsburgh, PA, USA

4 School of Medicine, Department of Medicine, University of Pittsburgh, Pittsburg, PA, USA

5 Spinal Cord Injury Service, Department of Veterans Affairs, VA Puget Sound Health Care System, Seattle, WA, USA
6 Department of Rehabilitation Medicine, University of Washington, Seattle, WA, USA

7 Department of Veterans Affairs, VA Salt Lake City Healthcare System, Salt Lake City, UT, USA

8 Division of Epidemiology, Department of Medicine, University of Utah, Salt Lake City, UT, USA

9 Department of Physical Medicine and Rehabilitation, Feinberg School of Medicine, Northwestern University, Chicago, IL, USA

10 Department of Preventive Medicine and Center for Health Care Studies, Institute for Public Health and Medicine, Feinberg School of Medicine, Northwestern University, Chicago, IL, USA 


\section{Introduction}

The Centers of Disease Control and Prevention (CDC) estimate approximately two million illnesses and 23,000 deaths are caused by antibiotic resistant bacteria every year [1] Specifically, multidrug resistant gram-negative organisms (MDRGNOs) have been a growing concern in healthcare and community settings [1]. These are particularly worrisome as MDRGNOs are often resistant to nearly all commonly used antibiotics. Gram-negative bacteria are frequently implicated in the most serious healthcare-associated infections (HAIs), with the common pathogens being Enterobacteriaceae, Pseudomonas aeruginosa, and Acinetobacter [1, 2].

Individuals with spinal cord injury or disorder (SCI/D) are at particularly high risk for infections due to frequent contact with the healthcare system, use of invasive medical devices, and antibiotic use [3-5]. Infections have been noted as a predictor of mortality and re-hospitalizations in individuals with SCI/D [6, 7]. In addition, previous research has found that individuals with SCI who have a hospitalacquired infection (HAI) have lowered survival [8].

Infections with MDRGNOs are associated with increased morbidity and mortality in general patient populations [9]. Gram-negative organisms are highly prevalent in individuals with SCI/D [10, 11], with increasing resistance over time [10]. Healthcare exposures, older age, exposure to antibiotics such as fluoroquinolones, carbapenems, cephalosporins, and sulfonamides, and comorbidities have been associated with MDRGNOs in those with SCI/D [10-13]. Furthermore, Fitzpatrick et al. found that although there were no differences in mortality rate, individuals with SCI/D colonized or infected with extended-spectrum $\beta$-lactamase (ESBL) bacteria had a longer postculture length of stay (LOS) than those with non-ESBL bacteria [5]. Additional research, focusing on MDR Acinetobacter in those with SCI/D did find increased risk of 30-day mortality in those with MDR cultures [13].

Therefore, the objective of this study was to assess the association between MDRGNOs and 1-year mortality, 30-day readmission, and postculture LOS in individuals with SCI/D stratified by case setting [inpatient, outpatient, long-term care (LTC)].

\section{Methods}

\section{Study design, setting, and population}

This was a retrospective cohort study of national Veterans Affairs (VA) medical encounter, microbiology, and pharmacy data from all 142 VA facilities. The first year of data (January 1, 2011 to December 31, 2011) was utilized to determine exposure for risk factors, and all microbiology culture data and analyses were based on the final 2 years of data collected January 1, 2012 to December 31, 2013. Veterans with SCI/D seen at a VA medical center during the study period were included. Veterans with multiple sclerosis and amyotrophic lateral sclerosis were excluded as these conditions are not associated with stable nonprogressive spinal cord neurological deficits and therefore not the focus of the VA SCI/D system of care.

\section{Data sources and definitions}

Several national VA datasets were utilized for this study. Patient demographics, healthcare utilization, facility information, microbiology, and pharmacy data were obtained from the VA Corporate Data Warehouse (CDW). The CDW is a national repository that is updated daily and includes clinical and administrative data from the Veterans Health Administration (VHA). The VA Vital Status File contains dates of death combined from the Veterans Benefits Administration Beneficiary Identification and Records Location System death file, the VA Medicare Vital Status File, and the Social Security Administration Death Master File, and was used to obtain mortality data. SCI/D characteristics were collected from the VA SCD registry which is a national database that contains spinal cord injury information derived from patient registries.

Demographic data (age, sex, race/ethnicity, and comorbidities) were identified 1 year prior to and during the visit or admission the culture was identified. Specimen type was categorized into blood, urine, sputum, and other (wound, tissue, body fluid, and bone cultures). ICD-9 codes from the Deyo-Charlson co-morbidity index [14], including pressure ulcer which is a common condition in SCI/D individuals, were used to identify comorbidities. Duration of SCI was categorized between $0-10$ years, 11-20 years, and 21+ years. Healthcare exposure was defined as exposures in the previous 90 days prior to culture date (long-term care, intensive care unit stay, surgery, mechanical ventilation, and previous healthcare admission). Antibiotic and steroid exposures were defined as receipt of the medication in the 90 days before culture date. The VA utilizes a hub and spoke system where 24 VA facilities are designated 'hubs' or SCI centers delivering specialized SCI care. 'Hub' centers are connected to 'spoke' facilities; spoke facilities provide community-based care for SCI/D individuals. Region was defined using the US Census Bureau regions; San Juan, Puerto Rico, and Manila, Philippines were grouped into the South region.

Multiple cultures from the same patient within 30 days were excluded as well as cultures that did not grow at least one gram-negative bacteria. Only the first culture per patient that grew a gram-negative bacteria during the study period was included. All gram-negative isolates were identified as MDR or non-MDR based on definitions from Magiorakos 


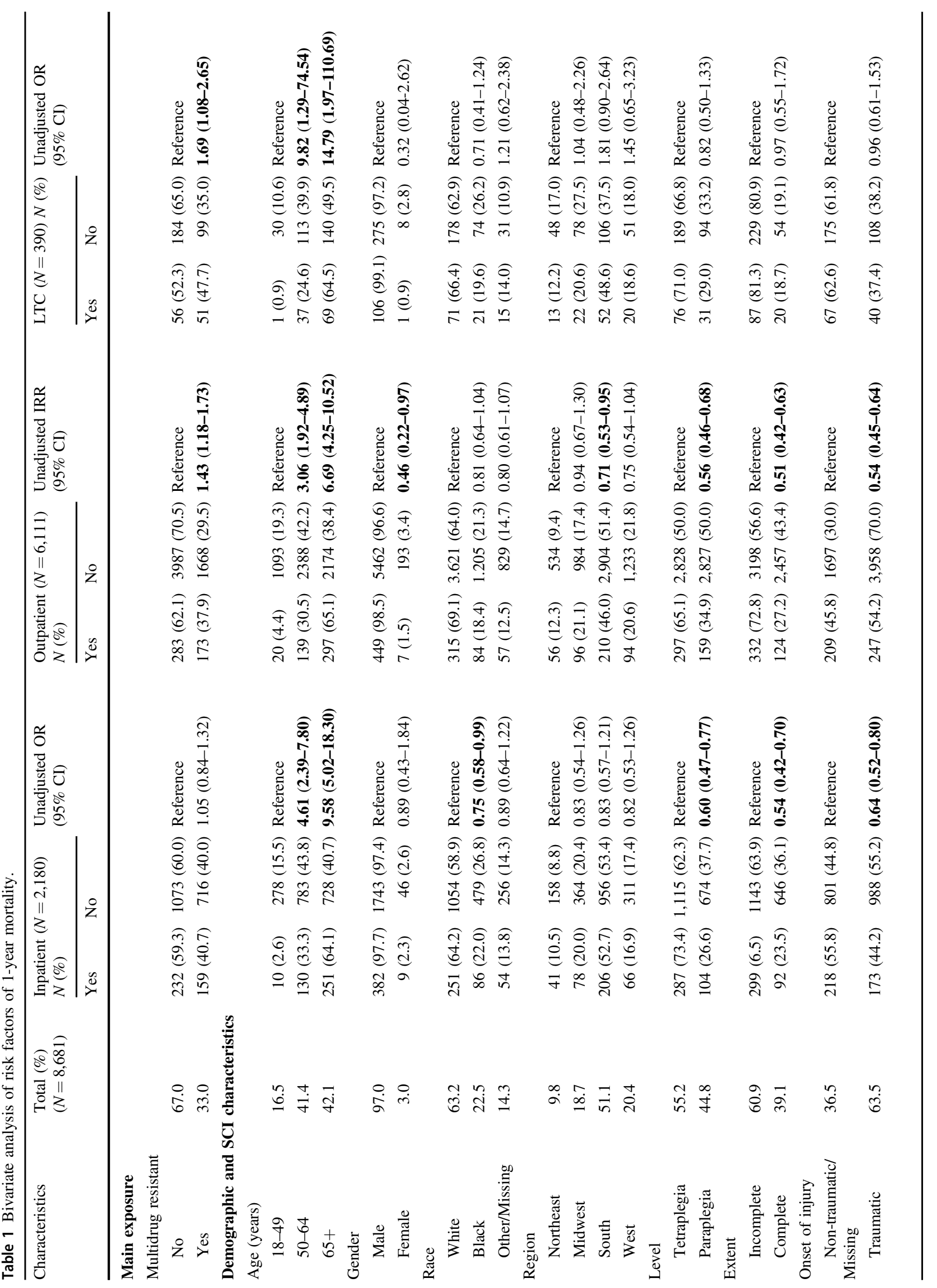




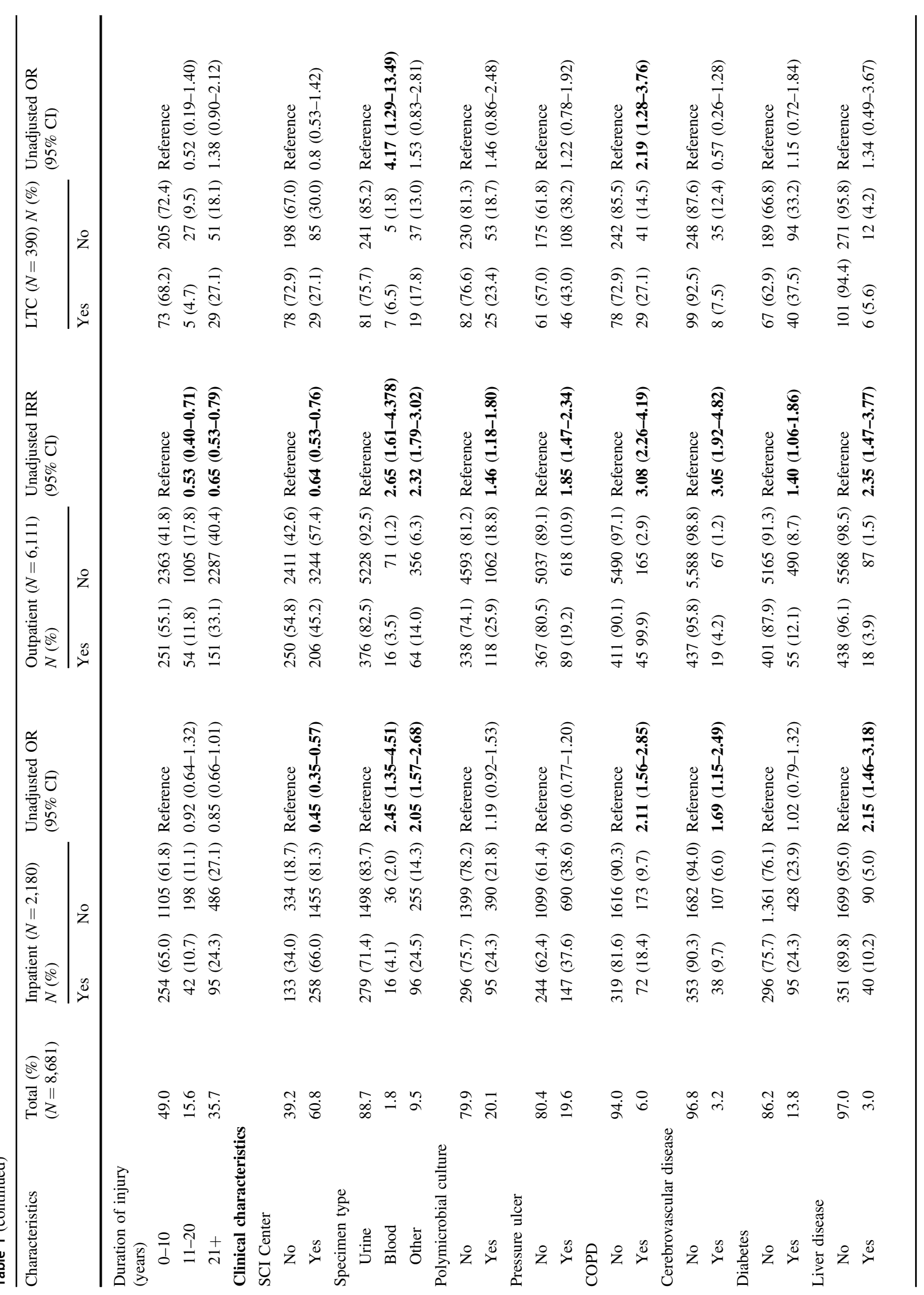




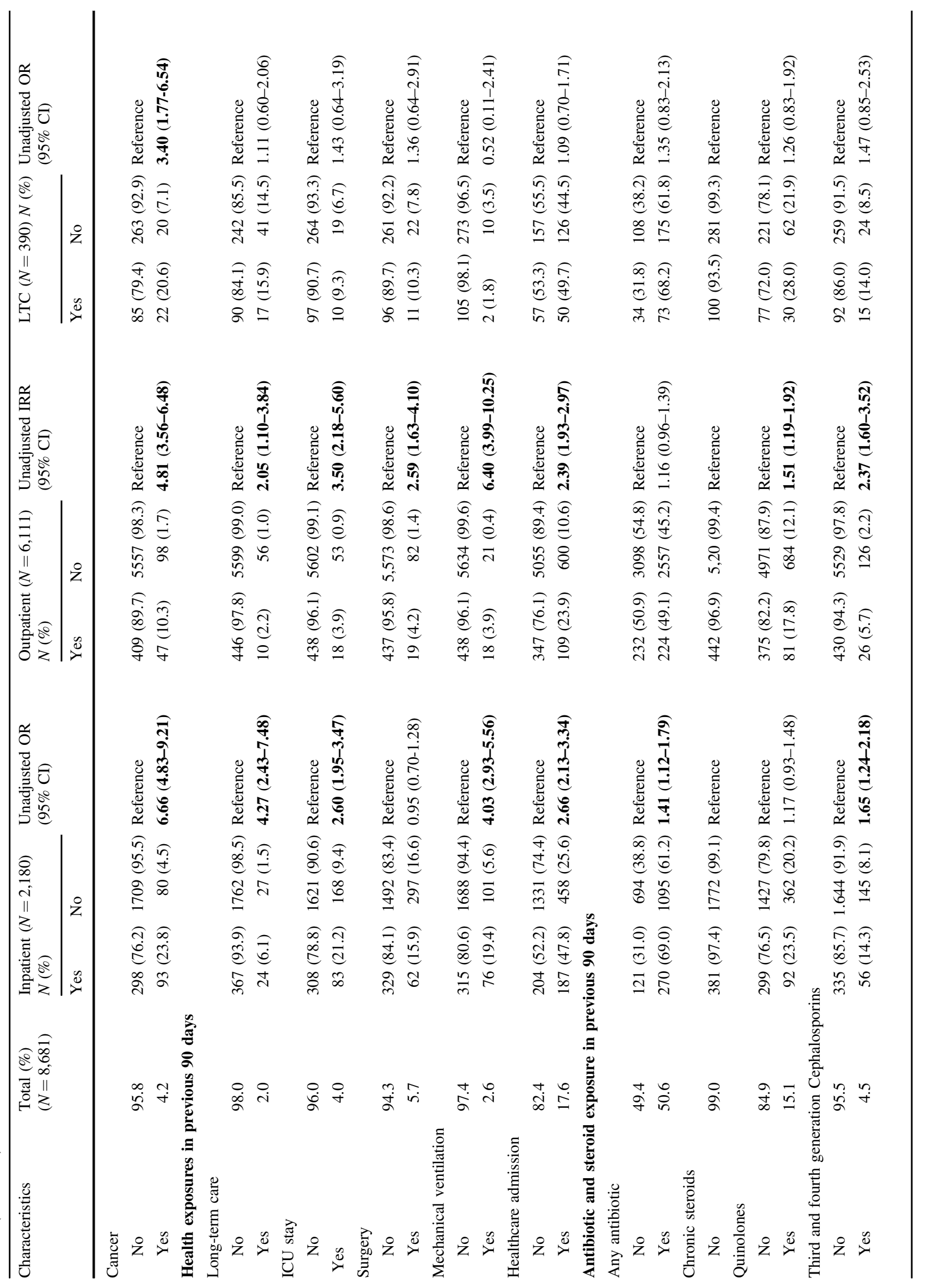




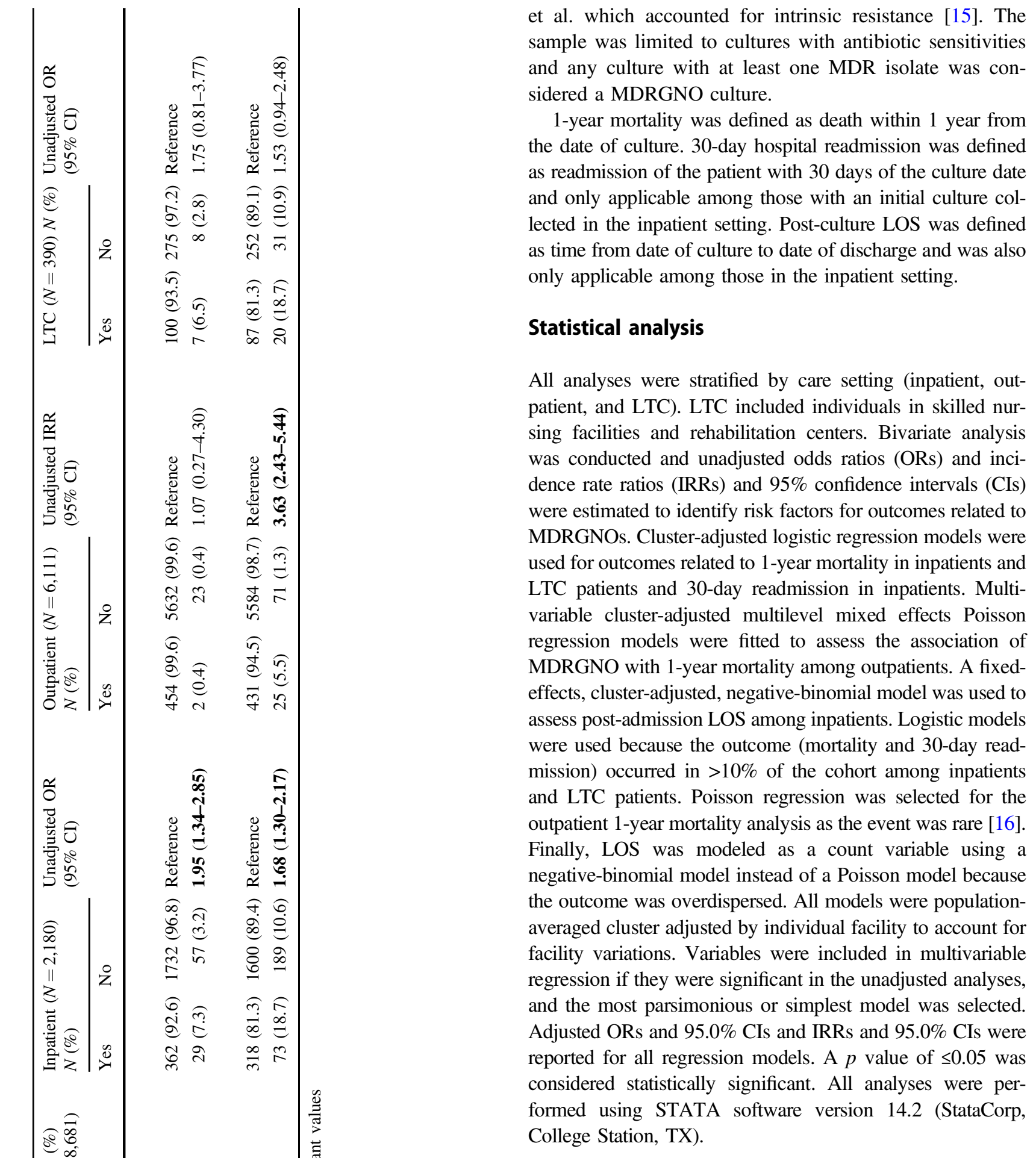

Results

Overview

There was a total of 8,681 individuals with SCI/D who had a gram-negative bacteria grow in culture during the study period. The sample had a mean age of $62.1(\mathrm{sd}=13.2)$ 


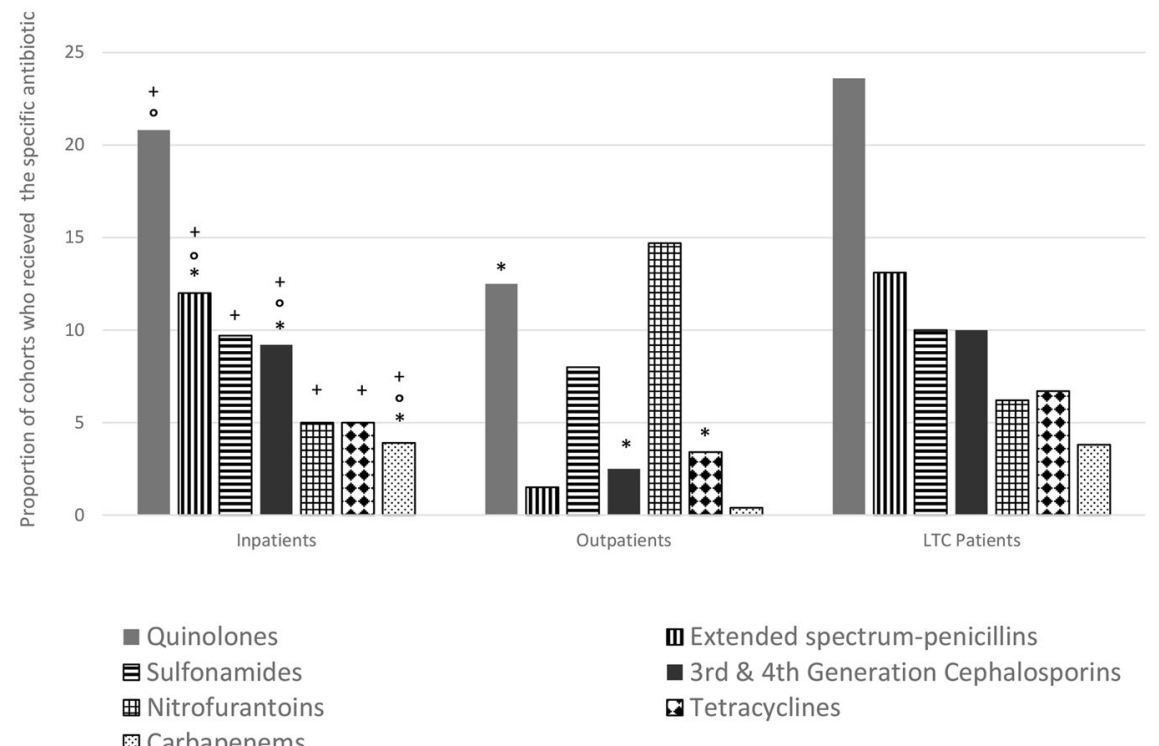

Fig. 1 Proportion of inpatients, outpatients, and LTC individuals that received the type of antibiotic 90 days before and association with outcomes of mortality, readmission, and length of stay. Asterisk denotes unadjusted OR or unadjusted IRR were significant and the proportion of those that received the specific antibiotic were different among those that died and did not die. White circle denotes unadjusted

years, SCI duration of $19.4(\mathrm{sd}=15.5)$ years, and Charlson co-morbidity score of $2.3(\mathrm{sd}=2.0)$. Most of the cohort was White (63.2\%), male (96.7\%), from the South (51.1\%), and about half the cohort had tetraplegia (55.2\%). Most specimens were urine cultures (88.7\%) and $20 \%$ of patients had a polymicrobial culture. The majority of patients were outpatients $(n=6111,70.4 \%)$. Inpatients comprised $25.1 \%$ of the total cohort $(n=2180)$ and the remaining were LTC patients $(n=390,4.5 \%)$. From the overall sample, 2866 of gram-negative cultures $(33.0 \%)$ were MDR. Overall, $40.0 \%$ $(n=875)$ of inpatient cultures, $30.1 \%(n=1,841)$ of outpatient cultures, and $38.4 \%(n=150)$ of LTC patient gramnegative cultures were MDR.

Overall, $11.0 \%$ of the cohort $(n=954)$ died within 1 year of culture date (13.4\% of the MDRGNOs versus $9.8 \%$ of non-MDRGNOs). Of those who died in 1 year, 456 (47.8\%) were outpatients, 391 (41.0\%) were inpatients, and 107 $(11.2 \%)$ were LTC patients. Overall, median postculture LOS in inpatients was $25(\mathrm{IQR}=82)$ days (MDRGNO [37; IQR: 111] vs non-MDRGNO [21; IQR: 71]). A total of 297 inpatients (13.6\%) experienced a readmission within 30 days of culture date (14.9\% of the MDRGNOs vs $12.9 \%$ of the non-MDRGNOs).

\section{Bivariate unadjusted results}

Bivariate unadjusted results for showed that having an MDRGNO was not associated with 1-year mortality in
OR was significant and the proportion of those that received the specific antibiotic were different among those that were readmitted and were not readmitted. Plus symbol denotes unadjusted IRR was significant and the length of stay differed for those that received the specific antibiotic compared with those that did not.

inpatients (OR: 1.15, 95\% CI, 0.87-1.52) (Table 1). However, having an MDRGNO was associated with increased 1-year mortality in outpatients (IRR: $1.28,95 \% \mathrm{CI}$, $1.06-1.54)$ and among LTC patients $(\mathrm{OR}=2.06,95 \% \mathrm{CI}$, 1.28-3.31). Among inpatients, risk factors for 1-year mortality included increased age, White race (vs. Black race), SCI characteristics, not being at an SCI center, blood or other specimen types (vs. urine), comorbidities, healthcare stay, and antibiotics or steroids in the previous 90 days before culture. For outpatients, similar risk factors were found for mortality; in addition male sex, north region (vs. south), increased duration of injury, having a polymicrobial culture, surgery, and mechanical ventilation were found to be risk factors for outpatients. Risk factors for LTC patients included increased age, having COPD or tumor or cancer, and use of chronic steroids. Individual exposure data in the previous 90 days is described in Fig. 1. The most frequent antibiotics prescribed were quinolones, extendedspectrum penicillins, and sulfonamides for inpatient and LTC groups. Among outpatients, the most frequently prescribed antibiotics were nitrofurantoins, quinolones, and sulfonamides. Carbapenems, third/fourth generation cephalosporins, and extended-spectrum penicillins increased the odds of inpatient mortality while quinolones, third/fourth generation cephalosporins, and extended-spectrum penicillins increased the risk of mortality in outpatients. No antibiotics were associated with increased risk of mortality in LTC individuals. 
Table 2 Bivariate analysis of risk factors of 30-day readmission and increased length of stay among inpatients ${ }^{\mathrm{a}}$.

\begin{tabular}{lllll}
\hline Characteristics & $\begin{array}{l}\text { Total }(\%) \\
(N=8681)\end{array}$ & $\begin{array}{l}\text { Inpatient 30-day readmission } \\
(N=2180) N(\%)\end{array}$ & $\begin{array}{l}\text { Unadjusted OR } \\
(95 \% \mathrm{CI})\end{array}$ & $\begin{array}{l}\text { Inpatient LOS } \\
\text { unadjusted IRR } \\
(95 \% \mathrm{CI})\end{array}$ \\
\cline { 2 - 3 } & Yes & & No
\end{tabular}

\section{Main exposure}

Multidrug resistant

$\begin{array}{lrrrrr}\text { No } & 67.0 & 167(56.2) & 1138(60.4) & \text { Reference } & \text { Reference } \\ \text { Yes } & 33.0 & 130(43.8) & 745(39.6) & 1.23(0.96-1.57) & \mathbf{1 . 3 8}(\mathbf{1 . 3 7 - 1 . 3 9 )}\end{array}$

Demographic and SCI characteristics

Age (years)

18-49

50-64

$65+$

Gender

Male

Female

Race

White

Black

Other/Missing

Region

Northeast

Midwest

South

West

Level

Tetraplegia

Paraplegia

Extent

Incomplete
Complete

Onset of injury

Non-traumatic/Missing

Traumatic

16.5

41.4

42.1

97.0

3.0

63.2

22.5

14.3

9.8

18.7

51.1

20.4

55.2

44.8

60.9

39.1

36.5

63.5

Duration of injury (years)

$\begin{array}{ll}0-10 & 49.0 \\ 11-20 & 15.6 \\ 21+ & 35.7\end{array}$

Clinical characteristics

SCI center

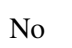

Yes

39.2

60.8

Specimen type

Urine

Blood

88.7

1.8

Other

9.5

Polymicrobial culture

No

79.9

Yes
$130(43.8)$

34 (11.5)

$140(47.1)$

123 (41.4)

288 (97.0)

9 (3.0)

173 (58.3)

$75(5.2)$

49 (16.5)

29 (9.8)

53 (17.9)

172 (57.9)

43 (14.4)

$191(64.3)$

106 (35.7)

$191(64.3)$

106 (35.7)

$136(45.8)$

$161(54.2)$

$180(60.6)$

34 (11.5)

83 (27.9)

83 27.9)

$214(72.1)$

$228(76.8)$

7 (2.4)

$62(20.9)$

$230(77.4)$

67 (22.6)

$\begin{array}{ll}254(13.5) & \text { Reference } \\ 773(41.0) & 1.35(0.91-2.02) \\ 856(45.5) & 1.07(0.72-1.61)\end{array}$

1837 (97.6) Reference

$46(2.4) \quad 1.25(0.60-2.58)$

$1132(60.1)$

Reference

$90(42.0)$

$261(13.9)$

$1.00(0.75-1.34)$

$1.22(0.87-1.73)$

$170(9.0)$

389 (20.7)

Reference

990 (52.6)

$0.80(0.49-1.30)$

$1.02(0.67-1.56)$

334 (17.7)

$0.75(0.46-1.25)$

1211 (64.3)

Reference

$672(35.7)$

$1.00(0.77-1.29)$

1251 (66.4)

Reference

632 (33.7)

$1.10(0.85-1.42)$

883 (46.9)

Reference

$1,000(53.1)$

1.05 (0.82-1.34)

1179 (62.6) Reference

206 (10.9)

1.08 (0.73-1.61)

498 (26.5)

$1.09(0.82-1.44)$

384 (20.4)

1499 (79.6)

Reference

$0.66(0.50-0.87)$

1549 (82.3)

Reference

45 (2.4)

$1.73(0.29-1.87)$

289 (15.4)

1.47 (1.09-1.87)

1465 (77.8)

Reference

1.06 (0.80-1.42)
Reference

1.04 (1.03-1.06)

1.02 (1.01-1.04)

Reference

0.81 (0.79-0.84)

Reference

$1.24(1.23-1.25)$

$0.75(0.74-0.76)$

Reference

$0.48(0.47-0.49)$

$0.52(0.51-0.53)$

$0.39(0.38-0.40)$

Reference

$0.93(0.92-0.94)$

Reference

1.18 (1.17-1.19)

Reference

1.03 (1.02-1.04)

Reference

1.20 (1.18-1.21)

$0.99(0.98-1.00)$

Reference

0.95 (0.94-0.96)

Reference

1.18 (1.15-1.21)

$1.04(1.03-1.05)$

Reference

$1.01(1.00-1.02)$ 
Table 2 (continued)

\begin{tabular}{|c|c|c|c|c|c|}
\hline \multirow[t]{2}{*}{ Characteristics } & \multirow[t]{2}{*}{$\begin{array}{l}\text { Total }(\%) \\
(N=8681)\end{array}$} & \multicolumn{2}{|c|}{$\begin{array}{l}\text { Inpatient 30-day readmission } \\
(N=2180) N(\%)\end{array}$} & \multirow[t]{2}{*}{$\begin{array}{l}\text { Unadjusted OR } \\
(95 \% \mathrm{CI})\end{array}$} & \multirow{2}{*}{$\begin{array}{l}\text { Inpatient LOS } \\
\text { unadjusted IRR } \\
(95 \% \text { CI })\end{array}$} \\
\hline & & Yes & No & & \\
\hline \multicolumn{6}{|l|}{ Pressure ulcer } \\
\hline No & 80.4 & $173(52.3)$ & $1,170(62.1)$ & Reference & Reference \\
\hline Yes & 19.6 & $124(41.7)$ & 713 (37.9) & $1.18(0.92-1.51)$ & $1.30(1.29-1.31)$ \\
\hline \multicolumn{6}{|l|}{ COPD } \\
\hline No & 94.0 & $259(87.2)$ & $1676(89.0)$ & Reference & Reference \\
\hline Yes & 6.0 & $38(12.8)$ & $207(11.0)$ & $1.19(0.82-1.72)$ & $0.77(0.75-0.78)$ \\
\hline \multicolumn{6}{|c|}{ Cerebrovascular disease } \\
\hline No & 96.8 & $275(92.6)$ & $1,760(93.5)$ & Reference & Reference \\
\hline Yes & 3.2 & $22(7.4)$ & $123(6.5)$ & $1.14(0.71-1.83)$ & $1.39(1.37-1.41)$ \\
\hline \multicolumn{6}{|l|}{ Diabetes } \\
\hline No & 86.2 & $228(76.8)$ & $1429(75.9)$ & Reference & Reference \\
\hline Yes & 13.8 & $69(23.2)$ & $454(24.1)$ & $0.95(0.71-1.27)$ & $0.82(0.81-0.83)$ \\
\hline \multicolumn{6}{|l|}{ Liver disease } \\
\hline No & 97.0 & $270(90.9)$ & $1780(9.5)$ & Reference & Reference \\
\hline Yes & 3.0 & $27(9.1)$ & $103(5.5)$ & $1.73(1.11-2.69)$ & $0.66(0.64-0.67)$ \\
\hline \multicolumn{6}{|l|}{ Cancer } \\
\hline No & 95.8 & $270(90.9)$ & $1737(92.3)$ & Reference & Reference \\
\hline Yes & 4.2 & $27(9.1)$ & $146(7.8)$ & $1.19(0.77-1.83)$ & $0.65(0.64-0.67)$ \\
\hline \multicolumn{6}{|c|}{ Health exposures in previous 90 days } \\
\hline \multicolumn{6}{|l|}{ Long-term care } \\
\hline No & 98.0 & $291(98.0)$ & $1838(97.6)$ & Reference & Reference \\
\hline Yes & 2.0 & $6(2.0)$ & $45(2.4)$ & $0.84(0.36-1.99)$ & $1.78(1.74-1.82)$ \\
\hline \multicolumn{6}{|l|}{ ICU stay } \\
\hline No & 96.0 & $254(85.5)$ & $1675(89.0)$ & Reference & Reference \\
\hline Yes & 4.0 & $43(14.5)$ & $208(11.0)$ & $1.36(0.96-1.94)$ & $0.79(0.78-0.80)$ \\
\hline \multicolumn{6}{|l|}{ Surgery } \\
\hline No & 94.3 & $244(82.2)$ & $1577(83.8)$ & Reference & Reference \\
\hline Yes & 5.7 & $53(17.9)$ & $306(16.2)$ & $1.11(0.81-1.54)$ & $0.75(0.74-0.76)$ \\
\hline \multicolumn{6}{|c|}{ Mechanical ventilation } \\
\hline No & 97.4 & $267(89.9)$ & $1736(92.2)$ & Reference & Reference \\
\hline Yes & 2.6 & $30(10.1)$ & $147(7.8)$ & $1.33(0.88-2.01)$ & $0.95(0.93-0.96)$ \\
\hline \multicolumn{6}{|c|}{ Healthcare admission } \\
\hline No & 82.4 & $184(62.0)$ & $1351(71.8)$ & Reference & Reference \\
\hline Yes & 17.6 & $113(38.0)$ & $532(28.2)$ & $1.56(1.21-2.01)$ & $0.79(0.78-0.80)$ \\
\hline \multicolumn{6}{|c|}{ Antibiotic and steroid exposure in previous 90 days } \\
\hline \multicolumn{6}{|c|}{ Any antibiotic } \\
\hline No & 49.4 & $92(31.0)$ & $723(38.4)$ & Reference & Reference \\
\hline Yes & 50.6 & $205(69.0)$ & $1160(61.6)$ & $1.39(1.07-1.81)$ & $0.88(0.87-0.89)$ \\
\hline \multicolumn{6}{|l|}{ Chronic steroids } \\
\hline No & 99.0 & $292(98.3)$ & $1,861(98.8)$ & Reference & Reference \\
\hline Yes & 1.0 & $5(1.7)$ & $22(1.2)$ & $1.45(0.54-3.85)$ & $1.21(1.17-1.25)$ \\
\hline \multicolumn{6}{|l|}{ Sulfonamides } \\
\hline No & 91.5 & $264(88.9)$ & 1705 (90.6) & Reference & Reference \\
\hline Yes & 8.5 & $33(11.1)$ & $178(9.5)$ & $1.17(0.81-1.68)$ & $1.39(1.37-1.41)$ \\
\hline
\end{tabular}


Table 2 (continued)

\begin{tabular}{|c|c|c|c|c|c|}
\hline \multirow[t]{2}{*}{ Characteristics } & \multirow[t]{2}{*}{$\begin{array}{l}\text { Total }(\%) \\
(N=8681)\end{array}$} & \multicolumn{2}{|c|}{$\begin{array}{l}\text { Inpatient 30-day readmission } \\
(N=2180) N(\%)\end{array}$} & \multirow[t]{2}{*}{$\begin{array}{l}\text { Unadjusted OR } \\
(95 \% \text { CI })\end{array}$} & \multirow{2}{*}{$\begin{array}{l}\text { Inpatient LOS } \\
\text { unadjusted IRR } \\
(95 \% \text { CI) }\end{array}$} \\
\hline & & Yes & No & & \\
\hline \multicolumn{6}{|l|}{ Nitrofurantoins } \\
\hline No & 94.0 & $284(95.6)$ & $1786(95.9)$ & Reference & Reference \\
\hline Yes & 6.0 & $13(4.4)$ & $97(5.1)$ & $0.86(0.49-1.50)$ & $0.95(0.92-0.97)$ \\
\hline \multicolumn{6}{|l|}{ Quinolones } \\
\hline No & 84.9 & $217(73.1)$ & $1,509(80.1)$ & Reference & Reference \\
\hline Yes & 15.1 & $80(26.9)$ & $374(19.9)$ & $1.40(1.08-1.81)$ & $0.96(0.95-0.97)$ \\
\hline \multicolumn{6}{|c|}{ Third and fourth generation Cephalosporins } \\
\hline No & 95.5 & $259(87.2)$ & $1720(91.3)$ & Reference & Reference \\
\hline Yes & 4.5 & $38(12.8)$ & $63(8.7)$ & $1.44(1.03-2.03)$ & $0.98(0.96-1.00)$ \\
\hline \multicolumn{6}{|l|}{ Carbapenems } \\
\hline No & 98.5 & $276(92.9)$ & $1818(96.6)$ & Reference & Reference \\
\hline Yes & 1.5 & $21(7.1)$ & $65(3.5)$ & $1.85(1.19-2.89)$ & $1.37(1.36-1.39)$ \\
\hline \multicolumn{6}{|c|}{ Extended-spectrum penicillins } \\
\hline No & 95.3 & $236(79.5)$ & $1,682(89.3)$ & Reference & Reference \\
\hline Yes & 4.7 & $61(20.5)$ & $201(10.7)$ & $1.89(1.43-2.51)$ & $0.76(0.76-0.78)$ \\
\hline \multicolumn{6}{|l|}{ Tetracyclines $^{\mathrm{b}}$} \\
\hline No & 96.0 & $278(93.6)$ & $1792(95.2)$ & Reference & Reference \\
\hline Yes & 4.0 & $19(6.4)$ & $91(4.8)$ & $1.29(0.81-2.05)$ & $1.31(1.29-1.33)$ \\
\hline
\end{tabular}

${ }^{a}$ Frequencies for LOS were not provided per risk factor as this was used as a continuous variable

${ }^{\mathrm{b}}$ Includes piperacillin, piperacillin/tazobactam, and ticarcillin/clavulanate

Bold typeset indicates significant values

Having an MDRGNO was not associated with increased 30-day readmission in inpatients (OR: 1.23, 95\% CI, 0.96-1.57) (Table 2). However, other factors associated with 30-day readmission in inpatients included not being at an SCI center, having a blood or other specimen (vs urine), having liver disease, and having a healthcare stay or antibiotic exposure in the previous 90 days before culture. Quinolones, third/fourth generation cephalosporins, carbapenems, and extended-spectrum penicillins exposure all increased risk of 30-day readmission (Fig. 1, Table 2).

In the bivariate analysis, MDR was associated with increased postculture LOS in inpatients (IRR: 1.38, 95\% CI, 1.37-1.39). Other risk factors for inpatients include male sex, Black race, northeast region, having tetraplegia or traumatic injury, increased duration of injury, other specimen types, having a pressure ulcer, COPD, cerebrovascular disease, diabetes, liver disease, tumor or cancer, not using chronic steroids, and having an LTC stay, not having surgery, not having mechanical ventilation, not having a genitourinary procedure, not having healthcare admission, or antibiotics in the previous 90 days before culture. Specific antibiotics associated with increased LOS were sulfonamides, carbapenems, and tetracyclines while nitrofurantoins, quinolones, and extended-spectrum penicillins were associated with a decreased LOS.

\section{Multivariable regression results}

In multivariable regression, after adjustment with other covariates, results showed that having an MDRGNO culture was not associated with 1-year mortality in inpatients (OR:1.15, 95\% CI, 0.87-1.52) but was associated with increased risk of 1-year mortality among outpatients (IRR: $1.28,95 \% \mathrm{CI}, 1.06-1.54)$ and increased odds of 1-year mortality in LTC patients (OR: 2.06, 95\% CI, 1.28-3.31) (Table 3). An MDRGNO culture was not associated with increased 30-day readmission in inpatients (OR: 1.25, 95\% CI, 0.97-1.61) but was associated with increased LOS among inpatients (IRR: 1.10, 95\% CI, 1.02-1.19) (Table 4).

\section{Discussion}

The purpose of this study was to understand how MDRGNOs impact outcomes in Veterans with SCI/D. Our results showed that having an MDRGNO culture was associated with 1-year mortality in outpatients and LTC 
Table 3 Multivariable regression analysis of 1-year mortality among inpatients, outpatients, and LTC patients.

\begin{tabular}{|c|c|c|c|}
\hline Variables & $\begin{array}{l}\text { Inpatients } \\
\text { OR }(95 \% \mathrm{CI}), p \text { value }\end{array}$ & $\begin{array}{l}\text { Outpatients } \\
\text { IIR ( } 95 \% \mathrm{CI}), p \text { value }\end{array}$ & $\begin{array}{l}\text { LTC patients } \\
\text { OR }(95 \% \mathrm{CI}), p \text { value }\end{array}$ \\
\hline \multicolumn{4}{|l|}{ Main exposure } \\
\hline MDR (Yes vs. No) & $1.15(0.87-1.52), 0.331$ & $1.28(1.06-1.54), 0.009$ & $2.06(1.28-3.31), 0.003$ \\
\hline \multicolumn{4}{|l|}{ Demographics and SCI characteristics } \\
\hline \multicolumn{4}{|l|}{ Age (years) } \\
\hline $50-64$ vs. $18-49$ & $3.42(1.71-6.83), 0.001$ & $2.69(1.72-4.21),<0.001$ & $8.13(0.98-67.68), 0.053$ \\
\hline $65+$ vs. $18-49$ & $7.02(3.74-13.19),<0.001$ & $5.46(3.45-8.62),<0.001$ & $13.87(1.53-125.63), 0.019$ \\
\hline \multicolumn{4}{|l|}{ Race } \\
\hline Black vs. White & $0.68(0.52-0.90), 0.006$ & $0.80(0.64-1.00), 0.05$ & - \\
\hline Other/Missing vs. White & $0.87(0.59-1.28), 0.485$ & $0.92(0.69-1.23), 0.563$ & - \\
\hline Level (Paraplegia vs. Tetraplegia) & $0.66(0.47-0.92), 0.014$ & $0.65(0.55-0.77),<0.001$ & \\
\hline \multicolumn{4}{|l|}{ Duration of Injury } \\
\hline $11-20$ vs $0-10$ & - & 0.83 (0.64-1.07), 0.155 & - \\
\hline $21+$ vs. $0-10$ & - & $0.79(0.64-0.97), 0.027$ & - \\
\hline \multicolumn{4}{|l|}{ Clinical characteristics } \\
\hline SCI center (Yes vs. No) & $0.71(0.54-0.93), 0.012$ & $0.69(0.58-0.82),<0.001$ & \\
\hline \multicolumn{4}{|l|}{ Specimen type } \\
\hline Blood vs. Urine & $1.52(0.75-3.08), 0.245$ & $1.34(0.87-2.05), 0.179$ & $5.09(1.18-21.88), 0.029$ \\
\hline Other vs. Urine & $1.60(1.22-2.10), 0.001$ & 1.49 (1.13-1.96), 0.004 & $1.69(0.83-3.42), 0.147$ \\
\hline Polymicrobial culture (Yes vs. No) & - & $1.38(1.09-1.74), 0.007$ & - \\
\hline Pressure ulcer (Yes vs. No) & - & $1.20(0.97-1.48), 0.098$ & - \\
\hline Charlson & $1.30(1.21-1.40),<0.001$ & $1.16(1.11-1.21),<0.001$ & $1.40(1.24-1.57),<0.001$ \\
\hline \multicolumn{4}{|c|}{ Healthcare exposures in previous 90 days } \\
\hline Long-term care (Yes vs. No) & $2.02(1.21-3.44), 0.007$ & - & - \\
\hline Mechanical ventilation (Yes vs. No) & $2.95(1.99-4.37),<0.001$ & $2.39(1.64-3.50),<0.001$ & - \\
\hline Healthcare exposure (Yes. vs. No) & $1.58(1.22-2.04),<0.001$ & $1.65(1.39-1.99),<0.001$ & - \\
\hline \multicolumn{4}{|c|}{ Antibiotic and steroid exposure in previous 90 days } \\
\hline Steroid Use (Yes vs. No) & -- & 1.97 (1.24-3.14), 0.004 & $16.07(4.29-60.16),<0.001$ \\
\hline
\end{tabular}

Blank spaces defined by "-_" indicate that those variables were not in the final model for that analysis

Bold typeset indicates significant values

patients. Furthermore, although MDR did not affect 30-day readmission among inpatients, it was significantly associated with increased LOS.

Overall, 1 in 10 Veterans with SCI/D with a gramnegative culture died within 1-year of the culture date. We found that outpatients with MDRGNOs had 27\% increased risk for 1-year mortality. Outpatients and LTC patients who died within 1-year of MDRGNO culture were older and sicker, with outpatients having had prior healthcare exposures. It is possible that outpatients with MDR may have substantial healthcare exposures, possibly for a chronic condition that may shorten life expectancy. However, our data showed that outpatients had a lower mean charlson score $(1.2(\mathrm{sd}=1.5))$ compared with inpatients $(3.0(\mathrm{sd}=$ 1.9)). It is unclear what is causing the increased risk for mortality in outpatients. It is also important to consider that MDRDNOs may be markers for increased mortality but may not be the cause of death. This is consistent with findings that those with increased risk for MDRGNOs were sicker and had prior healthcare exposures.

In addition, though we found no effect of 30-day readmission in inpatients, LOS for inpatients with MDRGNOs was significantly greater compared with those without MDRGNOs. Having an MDRGNO was associated with a $10.0 \%$ longer postculture LOS among inpatients in this study. Previously, having SCI has been found to be a unique predictor for increased LOS after HAI infection among those in a rehabilitation unit [17]. Furthermore, our results are also consistent with other published findings that nosocomial infections and MDR infection in those with SCI/D result in increased LOS $[8,18,19]$. However, as noted in the case of mortality, MDR may be a marker for increased LOS and may be a part of a combination of factors that increase postculture LOS. 
Table 4 Multivariable regression analysis of 30-day readmission and length of stay among inpatients.

\begin{tabular}{|c|c|c|}
\hline Variables & $\begin{array}{l}\text { Inpatients } \\
\text { 30-day readmission } \\
\text { OR }(95 \% \mathrm{CI}), p \text { value }\end{array}$ & $\begin{array}{l}\text { Length of stay } \\
\text { IIR }(95 \% \mathrm{CI}), p \text { value }\end{array}$ \\
\hline \multicolumn{3}{|l|}{ Main exposure } \\
\hline \multicolumn{2}{|l|}{ Demographics and SCI characteristics } & $1.10(1.02-1.19), 0.018$ \\
\hline \multicolumn{3}{|l|}{ Region } \\
\hline Midwest vs. Northeast & - & $1.04(0.85-1.26), 0.728$ \\
\hline South vs. Northeast & - & $1.15(0.96-1.38), 0.118$ \\
\hline West vs. Northeast & - & $1.16(0.95-1.42), 0.151$ \\
\hline \multicolumn{3}{|l|}{ Duration of Injury } \\
\hline $11-20$ vs $0-10$ & - & $0.82(0.73-0.94), 0.003$ \\
\hline $21+$ vs. $0-10$ & - & $0.81(0.74-0.89),<0.001$ \\
\hline \multicolumn{3}{|l|}{ Clinical characteristics } \\
\hline SCI center (Yes vs. No) & $0.69(0.49-0.96), 0.029$ & $1.46(1.27-1.66),<0.001$ \\
\hline \multicolumn{3}{|l|}{ Specimen type } \\
\hline Blood vs. Urine & - & $1.09(0.84-1.40), 0.520$ \\
\hline Other vs. Urine & - & $1.15(1.03-1.28), 0.011$ \\
\hline Cerebrovascular Disease (Yes vs. No) & & $1.21(1.04-1.41), 0.012$ \\
\hline Charlson & $0.96(0.91-1.01), 0.110$ & -- \\
\hline \multicolumn{3}{|l|}{ Healthcare exposures in previous 90 days } \\
\hline Long-term care (Yes vs. No) & & 1.53 (1.19-1.96), 0.001 \\
\hline Healthcare exposure (Yes. vs. No) & 1.53 (1.18-1.99), 0.001 & -- \\
\hline
\end{tabular}

Blank spaces defined by "-" indicate that those variables were not in the final model for that analysis Bold typeset indicates significant values
There were several limitations within this study. First, our study did not distinguish between infection and colonization, specifically with urinary colonization. This is an important limitation, as including patients who were only colonized rather than actively infected may have masked or blunted the actual association reported in this study. Second, we did not capture data from healthcare external to the VA which may bias results if there are unique characteristics associated with those that receive care outside the VA. Finally, because our study focused on Veterans with SCI/D who receive care at the VA and are mostly male, the results of this study may not be generalizable to other populations with SCI/D. This study however, has the advantage that it reports on data from the VHA, the single largest integrated healthcare system in the United States that is also the largest provider of care for patients with SCI/D.

In summary, among a large national cohort of individuals with SCI/D and cultures with gram-negative organisms, having an MDRGNO was associated with increased risk and increased odds for 1-year mortality among outpatients and LTC patients, respectively. Furthermore, among inpatients, having an MDRGNO was not associated increased postculture LOS but not 30-day hospital readmission. It is important for clinicians to be aware of the increased risk for poor outcomes in patients with SCI/D and MDRGNOs.
Results from this study support increased attention to antibiotic stewardship in SCI/D populations to prevent the development of antibiotic resistance in gram-negative bacteria and effective infection prevention strategies to prevent transmission of MDRGNOs.

\section{Data archiving}

The datasets generated during and/or analyzed during the current study are not publicly available as the sample size is too large to obtain informed consents and HIPAA authorizations for public discloser of the final study data containing PII and/or PHI and would be inconsistent with the IRB approved waiver of informed consent and waiver of HIPPA authorization that was approved.

Funding This work was supported by funding from the Veterans Health Administration, Office of Research and Development, Rehabilitation Research and Development Service SPIRE Award (grant no. B-1583-P). The views expressed in this article are those of the authors and do not necessarily reflect the policy of the Department of Veterans Affairs or the US government.

Author contributions CTE was the principal investigator and responsible for overseeing the project that the data were obtained from. $\mathrm{CT}$ also provided guidance and expertize in epidemiological methods and reviewing the manuscript. SR was responsible for data analysis 
and writing the manuscript. MAF and KJS provided helped with writing the discussion and providing infectious disease expertize. SPB and SLV provided spinal cord injury expertise related to the paper and findings. MMJ provided clinical experience with VA microbiology data and infectious disease expertize. All authors provided edits and revisions of the manuscript.

\section{Compliance with ethical standards}

Conflict of interest The authors declare that they have no conflict of interest.

Ethical statement This study was approved by the Edward Hines Jr. VA Hospital IRB. We certify that all applicable institutional and governmental regulations concerning the ethical use of human subjects data were followed during the course of this research.

Publisher's note Springer Nature remains neutral with regard to jurisdictional claims in published maps and institutional affiliations.

\section{References}

1. Centers for Disease Control and Prevention. Antibiotic resistance threats in the United States. Atlanta, Georgia: Centers for Disease Control and Prevention; 2013. https://www.cdc.gov/drugresistance/ threat-report-2013/pdf/ar-threats-2013-508.pdf. Accessed 7 Mar 2019.

2. Weiner LM, Webb AK, Limbago B, Dudeck MA, Patel J, Kallen $\mathrm{AJ}$, et al. Antimicrobial-resistant pathogens associated with healthcare-associated infections: summary of data reported to the National Healthcare Safety Network at the Centers for Disease Control and Prevention, 2011-2014. Infect Control Hosp Epidemiol. 2016;37:1288-301.

3. Montgomerie JZ. Infections in patients with spinal cord injuries. Clin Infect Dis. 1997;25:1285-90.

4. Evans CT, LaVela SL, Weaver FM, Priebe M, Sandford P, Niemiec S, et al. Epidemiology of hospital-acquired infections in veterans with spinal cord injury and disorder. Infect Control Hosp Epidemiol. 2008;29:234-42.

5. Fitzpatrick MA, Suda KJ, Safdar N, Goldstein B, Jones MM, Poggensee $\mathrm{L}$, et al. Unique risks and clinical outcomes associated with extended-spectrum betalactamase Enterobacteriaceae in veterans with spinal cord injury/disorder: a case-case-control study. Infect Control Hosp Epidemiol. 2016;37:768-76.

6. Rabadi MH, Mayanna SK, Vincent AS. Predictors of mortality in veterans with traumatic spinal cord injury. Spinal Cord. 2013;51: 784-8.

7. DeJong G, Tian W, Hsieh CH, Junn C, Karam C, Ballard PH, et al. Rehospitalization in the first year of traumatic spinal cord injury after discharge from medical rehabilitation. Arch Phys Med Rehabil. 2013;94:S87-97.

8. LaVela SL, Evans CT, Miskevics S, Parada JP, Priebe M, Weaver FM. Long-term outcomes from nosocomial infections in persons with spinal cord injuries and disorders. Am J Infect Control. 2007;35:393-400.

9. Nelson RE, Slayton RB, Stevens VW, Jones MM, Khader K, Rubin MA, et al. Attributable mortality of healthcare-associated infections due to multidrug-resistant gram-negative bacteria and methicillin-resistant staphylococcus aureus. Infect Control Hosp Epidemiol. 2017;38:848-56.

10. Fitzpatrick MA, Suda KJ, Safdar N, Burns SP, Jones MM, Poggensee $\mathrm{L}$, et al. Changes in bacterial epidemiology and antibiotic resistance among Veterans with spinal cord injury/disorder over the past 9 years. J Spinal Cord Med. 2018;41:199-207.

11. Evans CT, Fitzpatrick MA, Jones MM, Burns SP, Poggensee L, Ramanathan $\mathrm{S}$, et al. Prevalence and factors associated with multidrug-resistant gram-negative organisms in patients with spinal cord injury. Infect control Hosp Epidemiol. 2017; 38:1464-71.

12. Kale IO, Fitzpatrick MA, Suda KJ, Burns SP, Poggensee L, Ramanathan $\mathrm{S}$, et al. Risk factors for community-associated multidrug-resistant Pseudomonas aeruginosa in veterans with spinal cord injury and disorder: a retrospective cohort study. Spinal Cord. 2017;55:687-91.

13. Ramanathan S, Suda KJ, Fitzpatrick MA, Poggensee L, LaVela SL, Burns SP, et al. Multidrug-resistant Acinetobacter: Risk factors and outcomes in veterans with spinal cord injuries and disorders. Am J Infect Control. 2017;45:1183-9.

14. Charlson ME, Pompei P, Ales KL, MacKenzie CR. A new method of classifying prognostic comorbidity in longitudinal studies: development and validation. J Chronic Dis. 1987;40:373-83.

15. Magiorakos AP, Srinivasan A, Carey RB, Carmeli Y, Falagas ME, Giske CG, et al. Multidrug-resistant, extensively drug-resistant and pandrug-resistant bacteria: an international expert proposal for interim standard definitions for acquired resistance. Clin Microbiol Infect. 2012;18:268-81.

16. Agresti A. Categorical data analysis. 3rd ed. Hoboken, New Jersey: John Wiley \& Sons; 2013.

17. Mylotte JM, Graham R, Kahler L, Young BL, Goodnough S. Impact of nosocomial infection on length of stay and functional improvement among patients admitted to an acute rehabilitation unit. Infect Control Hosp Epidemiol. 2001;22:83-7.

18. Giske CG, Monnet DL, Cars O, Carmeli Y. ReAct-action on antibiotic resistance. clinical and economic impact of common multidrug-resistant gram-negative bacilli. Antimicrob Agents Chemo. 2008;52:813-21.

19. Patel G, Huprikar S, Factor SH, Jenkins SG, Calfee DP. Outcomes of carbapenem-resistant Klebsiella pneumoniae infection and the impact of antimicrobial and adjunctive therapies. Infect Control Hosp Epidemiol. 2008;29:1099-106. 